\title{
RSA : peut-on apprendre des expériences étrangères ?
}

Un bilan des travaux sur l'Earned Income Tax Credit et le Working Family Tax Credit

RSA: can we learn from foreign experiences? A survey of the literature on EITC and WFTC

\section{Fanny Mikol et Véronique Rémy}

\section{OpenEdition \\ Journals}

Édition électronique

URL : http://journals.openedition.org/travailemploi/1658

DOI : 10.4000/travailemploi.1658

ISSN : 1775-416X

Éditeur

DARES - Ministère du Travail

Édition imprimée

Date de publication : 15 décembre 2009

Pagination : 63-75

ISSN : 0224-4365

Référence électronique

Fanny Mikol et Véronique Rémy, «RSA : peut-on apprendre des expériences étrangères ? », Travail et Emploi [En ligne], 120 | octobre-décembre 2009, mis en ligne le 05 décembre 2011, consulté le 19 avril 2019. URL : http://journals.openedition.org/travailemploi/1658; DOI : 10.4000/travailemploi.1658 


\title{
RSA : peut-on apprendre des expériences étrangères? Un bilan des travaux sur I'EITC et le WFTC
}

\author{
Fanny Mikol (*), Véronique Rémy ${ }^{* *}$ )
}

\begin{abstract}
Les pays anglo-saxons recourent depuis plusieurs dizaines d'années aux crédits d'impôt avec un double objectif de redistribution et d'incitation à la reprise d'activité. L'Earned Income Tax Credit aux États-Unis et le Working Family Tax Credit au Royaume-Uni ont été largement étudiés: ils ont eu des effets sur la participation au marché du travail des populations éligibles, différents selon la configuration familiale. Alors que ces crédits visent à augmenter le revenu des ménages, ils peuvent cependant selon certaines études réduire le salaire des travailleurs éligibles et non éligibles. Les conclusions des études anglo-saxonnes peuvent être riches d'enseignements pour anticiper les conséquences sur l'offre de travail et les salaires de la composante "crédit d'impôt» du revenu de solidarité active (RSA-chapeau). Perçu par les travailleurs à bas revenus, ce dispositif, par sa forme, se rapproche davantage des crédits d'impôt anglo-saxons que la prime pour l'emploi.
\end{abstract}

Les mesures de crédit d'impôt(1) existent déjà depuis plusieurs dizaines d'années aux États-Unis, avec l'Earned Income Tax Credit (EITC), et au Royaume-Uni, avec le Working Families Tax Credit (WFTC, remplacé en 2003 par le Working Tax Credit (WTC)). Outre leur impact redistributif, ces crédits d'impôts, relativement élevés, ont également eu des effets sur la participation au marché du travail des populations qui en étaient éloignées. Sur ce sujet, la littérature économique est très riche.

En France, un tel mécanisme n'a vu le jour qu'en 2001 avec la prime pour l'emploi (PPE). L'instauration de cette prime visait au départ à inciter les individus sans emploi à rechercher un emploi à temps plein. Son efficacité reste encore discutée. Sur le plan redistributif, malgré les revalorisations successives depuis sa mise en œuvre, elle est moins ciblée sur les premiers déciles de revenus que ses homologues anglo-saxons, du fait notamment de l'exclusion des travailleurs à temps très partiel et de son assise sur les revenus individuels.

\footnotetext{
(*) INSEE. fanny.molkol@insee.fr

(**) DARES, département des politiques de l'emploi; veronique.remy@dares.travail.gouv.fr. Au moment de la rédaction de cet article, les auteurs travaillaient à la Mission «Analyse économique de la DARES, Ministère du travail, des relations sociales, de la famille, de la solidarité et de la ville, 39-43 quai André-Citroën 75015 Paris. Nous tenons à remercier Thomas Le Barbanchon, Benoît Heitz, Sylvie Le Minez ainsi que les deux rapporteurs anonymes de la revue pour leurs remarques constructives et leurs suggestions lors de la rédaction de cet article. Nous restons seules responsables des erreurs éventuelles qui pourraient subsister dans l'article.

(1) Un crédit d'impôt correspond à une prestation liée à l'exercice d'un emploi et peut être versée sous forme d'une subvention ou d'une réduction de l'impôt versé.
}

Son impact sur l'emploi demeure quant à lui incertain, peu d'études ayant été menées sur ce dispositif; les rares études ex post existant à ce sujet concluent à un impact très faible voire non significatif de la PPE sur le taux d'emploi (COCHARD, JunOD, 2008; Stancanelli, 2007). L'ensemble de ces études (2) portent néanmoins sur la PPE d'avant 2004, plus faible qu'aujourd'hui. Les importantes revalorisations de la PPE intervenues depuis (entre 2001 et 2008, son montant a doublé pour les salariés au Smic à temps plein et quadruplé pour ceux au Smic à temps partiel) sont encore trop récentes pour être évaluées.

Avec la volonté du gouvernement de rendre le travail plus incitatif dès la première heure travaillée, a été institué au $1^{\text {er }}$ juin 2009 un revenu de solidarité active (RSA). Le RSA se substitue aux minima sociaux pour les personnes sans emploi (RMI et API) et constitue un complément de revenu durable pour les personnes ayant un faible revenu (les «travailleurs pauvres»). Ce deuxième volet du dispositif, appelé «RSA-chapeau» est donc, à la différence de l'intéressement temporaire du RMI et de l'API, un mécanisme pérenne. Il s'adresse en particulier aux bénéficiaires de la PPE, mais sa construction tire certaines leçons de cette dernière: ce nouveau dispositif est davantage ciblé sur les travailleurs pauvres et prend mieux en compte les revenus du foyer et la présence d'enfants du fait de son caractère familialisé. Ses effets redistributifs attendus sont donc plus importants que ceux

(2) Voir également la revue de littérature sur la PPE réalisée par Stancanelli, Sterdyniak (2004). 
de la PPE, Bonnefoy, Buffeteau, Cazenave (2009) estimant ex ante une baisse du taux de pauvreté de 0,4 point supplémentaire liée à sa mise en œuvre par rapport à la PPE revalorisée. Le présent article complète leur étude en apportant des éléments d'analyse sur les effets attendus sur la participation et les salaires du RSA-chapeau. Ce RSA-chapeau est plus proche des crédits d'impôt américain et britannique que la PPE et pourrait en effet avoir également des effets sur l'emploi plus marqués que la PPE, même si le maintien conjoint des deux dispositifs risque d'altérer sa lisibilité et donc son efficacité.

Les résultats des réformes anglo-saxonnes, dont les évaluations sont bien plus nombreuses que celles de la PPE, peuvent ainsi apporter des enseignements dans la perspective de la mise en place du RSA. La revue de littérature que cet article propose permet de rappeler les principaux résultats des études menées sur les effets des crédits d'impôts sur l'offre de travail, les heures travaillées et les salaires. Elle peut être utile pour mieux anticiper les effets de l'introduction du RSA en France sur l'activité des différents types de public concernés (couples monoactifs, bi-actifs, parents isolés...) ainsi que sur leurs perspectives salariales.

\section{Caractéristiques du RSA et comparaison avec les crédits d'impôt anglo-saxons}

Le RSA vise à assurer, de manière pérenne, une garantie de revenu à son bénéficiaire. Pour les personnes sans emploi, cette garantie de revenu correspond à un revenu minimum, dont le montant varie en fonction de la composition du foyer et du nombre d'enfants à charge, équivalent à l'ancien revenu minimum d'insertion (RMI) ou à l'allocation parent isolé (API) pour les familles monoparentales. Pour les personnes en emploi, cette garantie est complétée par une fraction des revenus professionnels des membres du foyer. Le RSA est dégressif avec les salaires perçus par le foyer au taux de $38 \%$ (voir graphique 1). Le montant du RSA pour un foyer percevant un salaire de $w$ est donc le suivant:

$$
R S A=\text { revenu minimum }-0,38 * w
$$

Le revenu garanti total du foyer s'écrit alors:

$$
\begin{aligned}
& \text { revenu garanti }=R S A+w \\
= & \text { revenu minimum }+0,62 * w
\end{aligned}
$$

Le RSA correspond donc à la somme d'un revenu d'assistance (ou «RSA-socle», équivalent de l'ancien RMI, dégressif au taux de $100 \%$ avec le salaire) et d'un complément lié à l'activité (ou «RSA-chapeau», correspondant au surplus de prestation par rapport au précédent RMI). Cette décomposition est illustrée sur le graphique 1. La partie du RSA qui constitue une incitation à la reprise d'ac- tivité correspond en fait au RSA-chapeau: elle est attribuée à une part importante de bénéficiaires de la PPE (voir graphiques 2 et 3). Le RSA-chapeau est cependant moins avantageux pour les couples bi-actifs, avec ou sans enfants, que la PPE, calculée au niveau du foyer fiscal (et non au niveau du ménage comme l'est le RSA) et selon un barème individualisé(3), favorable aux ménages où les deux conjoints travaillent(4). La loi pour le RSA prévoit que le montant du RSA-chapeau réduise d'autant la PPE perçue, c'est-à-dire que seules les personnes pour lesquelles la PPE est plus élevée que le RSA-chapeau reçoivent une «PPE résiduelle». C'est notamment le cas des célibataires sans enfants et des couples bi-actifs, mais ce surplus devrait être progressivement revu à la baisse pour ce type de configuration familiale avec la non-revalorisation de la PPE.

\section{Graphique 1 : Revenu garanti avec le RSA dans le cas d'un célibataire sans enfant}

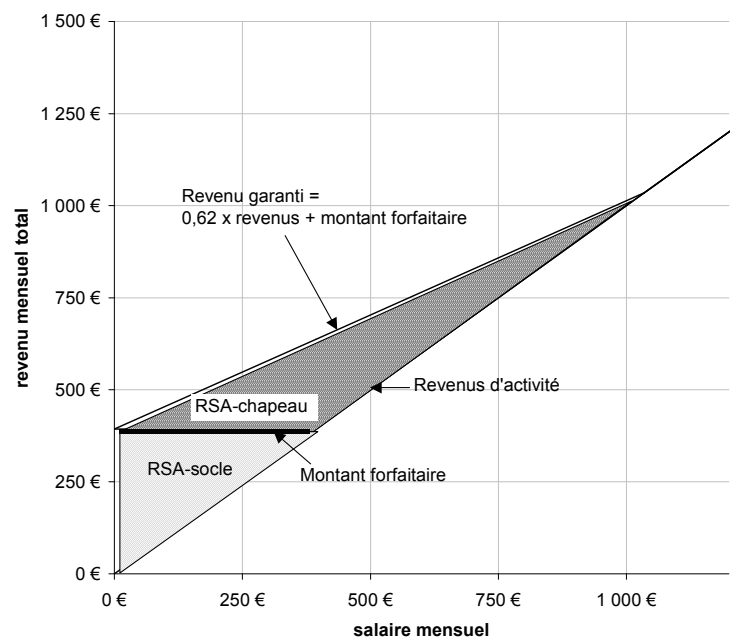

Sources: loi RSA, calculs DARES

Le revenu mensuel total correspond au salaire augmenté du RSA.

(3) Dans le cas de la PPE, les conditions de ressource au niveau du foyer fiscal sont peu restrictives par rapport aux conditions de revenus individuelles, plus contraignantes.

(4) En particulier, ce mode de calcul est favorable aux couples non mariés ou pacsés, qui constituent deux foyers fiscaux indépendants. 


\section{Graphique 2 : Célibataire sans enfant}

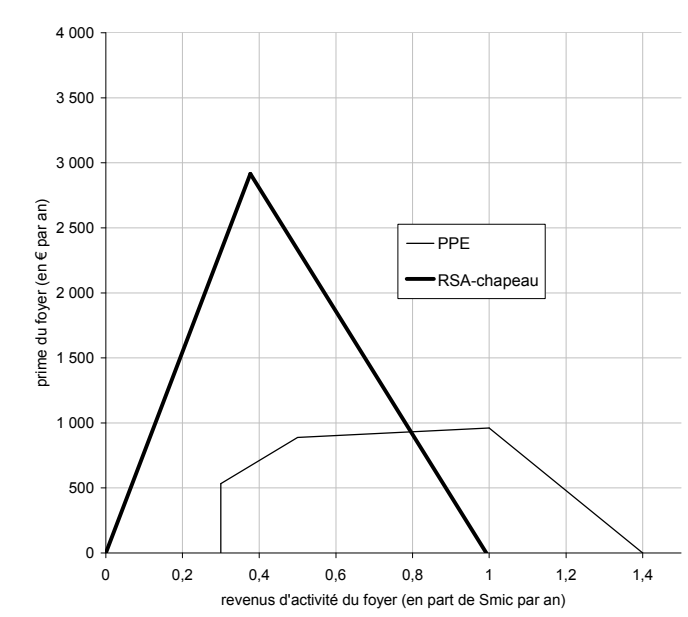

Source : Direction Générale des Impôts, calculs Dares.

Note : PPE en vigueur en 2008 (au titre des revenus2007).

Ces mesures d'incitation à la reprise d'activité sont relativement récentes en France au regard de leurs homologues anglo-saxonnes, dont les bases furent posées il y a pratiquement quarante ans. Des crédits d'impôt furent ainsi introduits dès 1975 aux USA et dès 1971(5) au Royaume-Uni, avec pour objectif de lutter contre la pauvreté infantile. L'EITC américain a par la suite été recentré pour rendre le travail plus attractif financièrement et encourager les individus percevant des revenus d'assistance à chercher un emploi. Le WFTC britannique mis en place en 1999, en remplacement du Family Credit $(F C)$, avait comme double objectif d'inciter à la reprise d'un emploi et d'encourager les personnes faiblement rémunérées à progresser dans l'échelle des salaires. Il s'agissait de soutenir les revenus des familles actives avec enfants, auxquelles le WFTC était exclusivement destiné, contrairement au Working Tax Credit (WTC) qui remplace le WFTC depuis 2003. Le $W T C$ bénéficie ainsi également aux personnes sans enfant à condition que ces dernières exercent une activité de plus de 30 heures hebdomadaires, mais il reste nettement plus élevé pour les familles avec enfants.

L'EITC et le WTC dépendent des revenus du foyer et de la configuration familiale. Ils bénéficient majoritairement à des parents isolés et concernent essentiellement les premiers déciles de revenus (Delarue

(5) À l'origine le Family Income Support (FIS), voir Delarue (2000).

\section{Graphique 3 : Couple bi-actif sans enfants, avec un des conjoints au Smic temps plein}

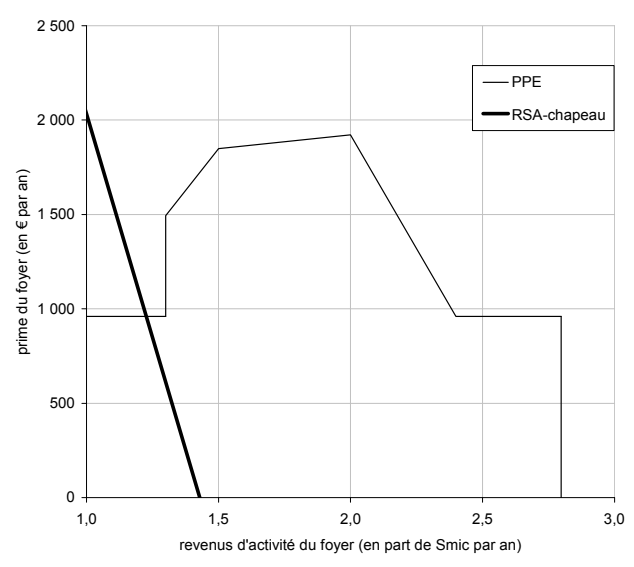

Source : Direction Générale des Impôts, calculs Dares. Note : PPE en vigueur en 2008 (au titre des revenus 2007).

(2000), Eissa et Hoynes (2005)) (6). Si le WTC est plus élevé que l'EITC pour les familles avec enfant, il entre dans le calcul d'autres prestations (Housing Benefit, Income Support) ce qui minore in fine son effet sur le revenu disponible. Les seuils d'éligibilité de l'EITC et du WTC diffèrent: respectivement dès la première heure travaillée et au minimum 16 heures par semaine ( 30 heures pour les personnes seules, soit une incitation ciblée sur la reprise d'un emploi à temps complet pour ce public(7)). Enfin, ces mesures consistent aujourd'hui en des crédits d'impôt versés annuellement au salarié. Avant 2005 cependant, le WTC - comme le WFTC - était versé mensuellement directement par l'employeur via la fiche de paie ce qui présentait certains risques (voir infra).

Les illustrations par des cas-types permettent une première comparaison entre le nouveau dispositif français et les mesures américaines et britanniques actuelles (voir graphiques 4 à 6). L'EITC comprend une phase d'entrée où il augmente proportionnellement au revenu (taux d'entrée de $40 \%$ en 2008 pour les familles avec deux enfants ou plus (34\% avec un enfant)), puis une phase de plateau où il est maximum et une phase de sortie où il diminue pour s'annuler au seuil d'extinction (taux de sortie de $16 \%(21 \%))$. Le $W T C$ commence par une phase

(6) Le ciblage sur le $2^{\mathrm{e}}$ et $3^{\mathrm{e}}$ décile de revenu s'explique d'autant plus pour le WFTC qu'il ne s'applique qu'à partir de la 16ème heure travaillée. Le $1^{\text {er }}$ décile est moins touché dans la mesure où il concentre davantage des individus hors de l'emploi. (7) Il est également majoré au-delà de ce seuil pour les familles avec enfants. 
de plateau, puis décroît au-delà d'un certain seuil de revenu (au taux de 39\%). Enfin le RSA-chapeau comprend une phase d'entrée (au taux de 62\%) et une phase de sortie (au taux de $38 \%$ ).

Le RSA-chapeau est perçu par les personnes en emploi dès le premier euro gagné, et est donc ciblé sur les premiers déciles de revenus. En cela, il s'agit d'un dispositif favorable au travail à temps partiel, et notamment aux très faibles durées de travail, comme l'EITC qui atteint son maximum aux alentours du mi-temps mais contrairement au Working Tax Credit (WTC) qui ne se déclenche qu'à partir de 16 heures de travail hebdomadaires (en deçà de ce seuil sont cependant versés le Child Tax Credit (CTC) - pour les familles avec enfants, ainsi qu'une partie de l'Income Support).

Pour les familles avec enfants, le niveau du RSA est intermédiaire entre celui du WTC (auquel s'ajoute le CTC) et de l'EITC. Il est davantage ciblé sur les plus bas déciles de revenus. À noter qu'à l'image du WTC qui entre dans le calcul des autres prestations, le RSA est réduit du montant des autres prestations perçues (allocations familiales...). Par ailleurs, alors que l'EITC et le WTC demeurent surtout des dispo-

\section{Graphique 5 : Célibataire avec un enfant}

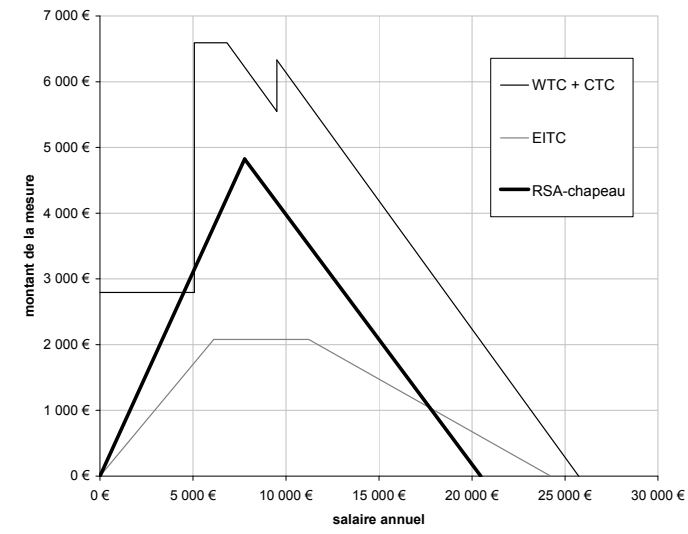

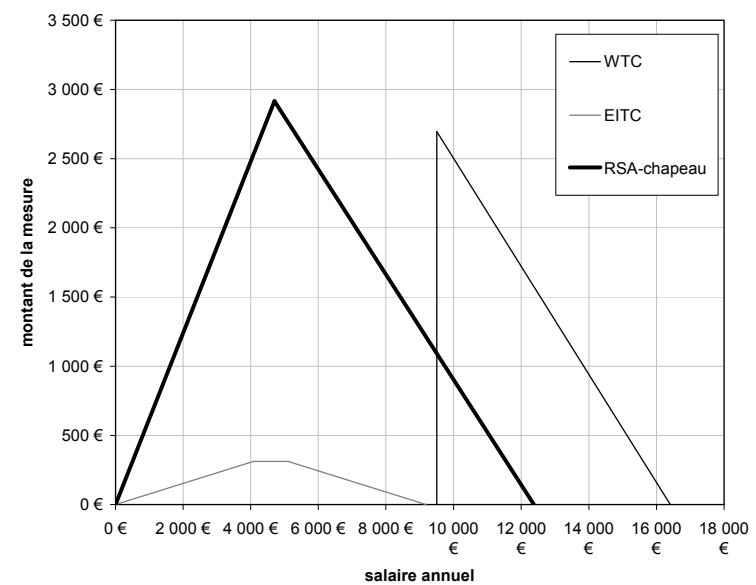

Sources: Inland Revenue (Royaume-Uni) ; Internal Revenue Service (USA); loi RSA ; calculs Dares (France).

Notes : Les barèmes de l'EITC et du WFTC correspondent à ceux en vigueur en 2008. L'unité de compte est l'euro, les montants étant exprimés en parité de pouvoir d'achat de la consommation privée en 2008

\section{Graphique 6: Célibataire avec deux enfants}

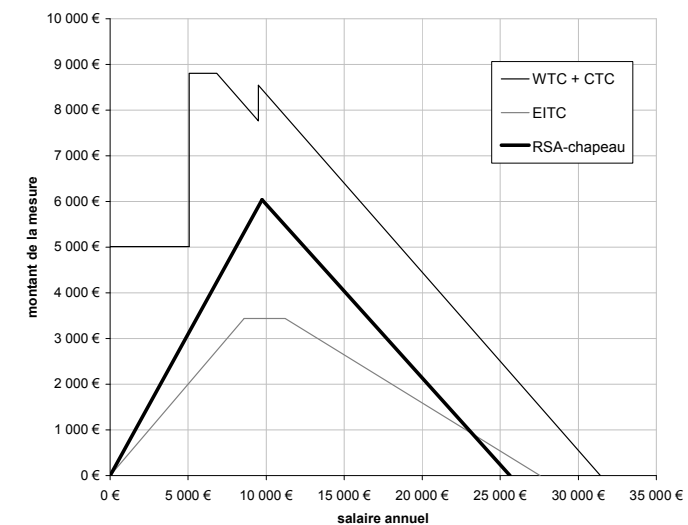

Sources : Inland Revenue (Royaume-Uni); Internal Revenue Service (USA); loi RSA ; calculs Dares (France).

Notes : le WTC est ici représenté avec le CTC (versé également aux personnes sans emploi, et dégressif à partir du seuil d'extinction du WTC), qui est perçu par les familles avec enfants. L'ensemble de ces prestations familialisées sont identiques pour un couple et pour un parent isolé. Les barèmes de l'EITC et du WFTC correspondent à ceux en vigueur en 2008. L'unité de compte est l'euro, les montants étant exprimés en parité de pouvoir d'achat de la consommation privée en 2008.

sitifs favorables aux familles pauvres avec enfants, le RSA reste relativement élevé même pour les foyers sans enfant des premiers déciles de revenu (graphique 4).

L'introduction du WTC étant trop récente pour avoir été suffisamment évaluée, la revue de littérature se concentre, dans le cas anglais, sur le WFTC. 
Tableau 1 : Résultats des études sur les effets de l'EITC sur l'activité des mères isolées

\begin{tabular}{|l|l|l|l|}
\hline \multicolumn{1}{|c|}{ Étude } & \multicolumn{1}{|c|}{ Période } & \multicolumn{1}{c|}{ Champ } & \multicolumn{1}{c|}{ Méthode d'évaluation et résultat } \\
\hline EISSA, LieBman (1996) & Réforme de 1986 & Mères isolées & $\begin{array}{l}\text { Doubles différences: effet sur le taux d'emploi de } \\
+2,4 \text { points }\end{array}$ \\
\hline MEYER, RoSENBAUM $(1999,2000,2001)$ & $1984-1996$ & Mères isolées & $\begin{array}{l}\text { Doubles différences: effet positif sur le taux } \\
\text { d'emploi }\end{array}$ \\
\hline LoONEY (2005) & $1993-1999$ & Mères isolées & $\begin{array}{l}\text { Doubles différences: 1'EITC explique 22\% de la } \\
\text { hausse du taux d'emploi }\end{array}$ \\
\hline RothSTEIN (2005) & Réforme de 1993 & Mères isolées & $\begin{array}{l}\text { Doubles différences, selon le salaire: effet positif, } \\
\text { mais décroissant avec le salaire. EITC explique } \\
\text { 26\% de la hausse du taux d'emploi entre 1992 } \\
\text { et 1995 }\end{array}$ \\
\hline
\end{tabular}

\section{Les principaux résultats des évaluations sur la participation au marché du travail}

\section{Les effets des mesures sur les parents isolés}

Les principaux résultats des études empiriques mesurant l'effet des différentes réformes(8) de l'EITC sur le taux d'emploi des mères isolées sont résumés dans le tableau 1 . La plupart font appel à la méthode des doubles différences qui consiste, dans les grandes lignes, à comparer l'évolution d'un groupe test (bénéficiant de la mesure) à un groupe de contrôle (n'en bénéficiant pas). EIsSA et LiEBMAN (1996) constatent ainsi que le taux d'activité des femmes célibataires avec enfants a augmenté de 2,4 points après la réforme de 1986 tandis que celui des femmes célibataires sans enfant ne s'est pas modifié. Néanmoins, ces dernières avaient déjà un taux de participation élevé ( $95,2 \%$ initialement contre $72,9 \%$ pour les premières) ce qui peut conduire à surestimer l'effet de la mesure puisque la marge d'augmentation de leur taux d'activité est plus faible même si la conjoncture s'améliore (BLUNDELL et WALKER (2002)). Toutefois, le résultat subsiste lorsque les auteurs se restreignent au segment des femmes peu qualifiées dont le taux d'activité est plus faible.

Meyer et Rosenbaum $(1999,2000,2001)$ étudient plusieurs réformes successives ayant revalorisé et étendu l'EITC et aboutissent au même résultat, quel que soit le groupe de contrôle retenu (femmes sans enfant, mères mariées...). Les différentes réformes auraient en effet conduit à une augmentation relative du taux d'emploi des mères isolées de 7,3 points par rapport aux célibataires sans enfants, cet effet étant principalement concentré sur la période après

(8) Trois grandes réformes de l'EITC ont eu lieu en 1986, en 1990 et en 1993. Ces trois réformes ont toutes consisté à accroître le niveau du crédit d'impôt, la seconde ayant consisté à augmenter spécifiquement le crédit pour les familles de deux enfants ou plus et la dernière, également la plus ambitieuse, ayant étendu le seuil d'éligibilité pour ces familles.
1990. Pour isoler l'impact de l'EITC, les auteurs ont ensuite estimé un modèle structurel d'offre de travail, tenant compte des autres réformes survenues entre 1984 et 1996(9). L'EITC expliquerait ainsi $60 \%$ de l'augmentation du taux d'emploi des mères isolées sur la sous-période 1986-1996 et 35\% sur la période 1992-1996.

Selon LOONEY (2005), l'EITC aurait contribué à $22 \%$ de la hausse de l'emploi des mères isolées entre 1993 et 1999 et à $23 \%$ de la baisse du nombre de prises en charge dans le domaine social. Cette étude comme la précédente tient compte d'un grand nombre de programmes sociaux mis en œuvre ou étendus en même temps que les différentes réformes de l'EITC. Pour identifier l'effet de ces dernières réformes, l'auteur n'a pas construit de modèle structurel, contrairement à MEYER et RoSENBAUM, mais a identifié plusieurs groupes de traitement et de contrôle selon différents critères: le moment auquel le programme intervient, le fait que les changements de barème affectent différemment les familles selon leur nombre d'enfants, ou encore la discontinuité des règles d'éligibilité, basées sur l'âge des enfants pour certains programmes. RothsteIN (2005) a étudié la réforme de 1993 en différenciant ses conséquences selon le niveau de salaire. Il constate que son effet relatif sur le taux d'emploi des mères célibataires (avec un ou deux enfants et plus) par rapport aux femmes célibataires sans enfant diminue avec le niveau de salaire.

Un certain nombre d'études récentes ont également utilisé la méthode des doubles différences pour évaluer l'impact de la mise en place du WFTC(10) (voir tableau 2). Selon Blundell, Brewer, ReEd et SHEPHARD (2005) qui comparent les mères isolées avec les femmes sans enfants, l'introduction du WFTC aurait entraîné une hausse de 3,6 points du

(9) En effet, les réformes de l'EITC se sont accompagnées d'autres réformes visant à encourager la participation au marché du travail dans un pays où les prestations sociales en cas d'inactivité demeurent très faibles (pour un état des lieux des réformes voir PÉRIVIER (2007)).

(10) Le WFTC a remplacé le Family Credit $(F C)$ en 1999: il garde la même forme mais est beaucoup plus élevé et est versé directement à l'employeur contrairement au FC. 
Tableau 2: Les évaluations du WFTC sur l'activité des parents isolés

\begin{tabular}{|l|l|l|l|}
\hline \multicolumn{1}{|c|}{ Étude } & \multicolumn{1}{|c|}{ Période } & \multicolumn{1}{c|}{ Champ } & \multicolumn{1}{c|}{ Méthode d'estimation et résultat } \\
\hline Blundell, Brewer et Shephard (2005) & Réforme de 1999 & Parents isolés & $\begin{array}{l}\text { Doubles différences : effet positif sur le taux } \\
\text { d'emploi: }+3,6 \text { points effet global + 25 000 à } \\
+60000 \text { emplois }\end{array}$ \\
\hline Francesconi, Van der Klauw (2004) & $1991-2001$ & Mères isolées & $\begin{array}{l}\text { Doubles différences: effet positif sur le taux } \\
\text { d'emploi: }+7 \text { points } \\
\text { Effet positif sur le taux de maintien dans l'emploi }\end{array}$ \\
\hline Gregg, Harkness (2003) & $1992-2002$ & Mères isolées & $\begin{array}{l}\text { Doubles différences: effet positif sur le taux } \\
\text { d'emploi: }+5 \text { points }\end{array}$ \\
\hline Paull et al. (2000), Preston, Walker (1999) & Réforme de 1999 & Mères isolées & $\begin{array}{l}\text { Modèle structurel: effet positif sur le taux } \\
\text { d'emploi: }+3,1 \text { points }\end{array}$ \\
\hline
\end{tabular}

taux d'emploi des premières. La hausse du taux d'emploi des mères isolées avec plusieurs enfants à charge ou avec un très jeune enfant serait plus importante dans la mesure où elles bénéficient d'un crédit d'impôt plus conséquent(11). Les auteurs estiment un impact global positif de la mesure sur l'emploi de 25000 à 60000 emplois, mais relativisent ces résultats en soulignant que la méthode utilisée ne permet pas de distinguer l'effet propre du WFTC des autres modifications concomitantes de taxes et d'allocations (12). En particulier, les mesures du type revalorisation du revenu minimum, susceptibles de réduire les incitations à la reprise d'emploi, ont pu amener à sous-estimer les effets du WFTC. En estimant des modèles structurels, PaUll, Walker et Zhu (2000) et Preston et Walker (1999) concluent que le taux d'activité des mères isolées s'est accru de plus de 3 points suite à la mise en place du WFTC.

D'autres études aboutissent à la même conclusion mais avec des ordres de grandeur plus élevés sur les taux de participation des mères isolées en utilisant toujours comme groupe de contrôle les femmes sans enfant: +7 points pour FrANCESCONI et Van Der Klaw (2004), +5 points pour GregG et HARKNESS (2003), dont la plus grande part provient de reprises d'emploi de plus de 30 heures. Ces différences sont notamment liées au fait que ces deux études étudient une période plus longue qui comprend, outre l'introduction du WFTC, les revalorisations du $F C$ intervenues en 1992 (abaisse-

(11) Le niveau relativement élevé du WFTC pour ces femmes ayant de jeunes enfants et/ou plusieurs enfants est suffisant pour compenser la traditionnelle moindre participation de ces femmes au marché du travail (provenant notamment des plus grandes difficultés en termes de modes de garde). Plusieurs études ont en effet mis en évidence que l'élasticité de l'offre de travail des mères par rapport aux incitations financières à l'emploi était d'autant plus faible que les enfants étaient jeunes et/ou nombreux (Brewer et al. (2003), Laroque, Salanié (2000)).

(12) En effet, contrairement à l'EITC, la réforme du WFTC s'est accompagnée de certaines mesures n'encourageant pas la reprise d'un emploi (différentes mesures d'augmentation des aides sociales). Cette réforme s'est inscrite dans le cadre du New Deal. Enfin, un salaire minimum a été instauré en même temps que le WFTC (pour une analyse détaillée des réformes au Royaume-Uni voir Delarue (2000)). ment du seuil d'entrée à 16 heures hebdomadaires travaillées) et 1995 (introduction d'une prime pour les reprises d'emploi de plus de 30 heures hebdomadaires). Par ailleurs, Francesconi et VAN Der KLAW (2004) se restreignent aux emplois de plus de 16 heures (c'est-à-dire uniquement ceux ouvrant droit au WFTC), ce qui explique un effet sur le taux d'emploi légèrement plus élevé que celui de GREGG et HARKNESS (2003).

Francesconi et Van Der Klaw (2004) constatent également que le taux de maintien dans l'emploi (c'est-à-dire la probabilité d'être toujours en emploi un an après la reprise d'un emploi) a augmenté d'environ 7 points entre 1991 et 2001. La mesure a eu un effet d'anticipation: un certain nombre d'individus, anticipant dès 1998 un gain plus important en cas de reprise d'une activité avec le $W F T C$, sont entrés sur le marché du travail avant que la mesure ne soit effective. Cet effet aurait contribué à +4 points de la hausse du taux d'emploi des mères isolées.

Les différentes études menées sur l'EITC et le WFTC mettent donc en évidence un effet positif des différentes réformes sur le taux d'emploi des parents isolés et concluent à une certaine efficacité des incitations financières à la reprise d'activité. Il est ainsi possible d'anticiper que le RSA aura lui aussi un effet favorable sur l'activité des parents isolés, qui occupent fréquemment un emploi à temps partiel pour lequel le RSA est relativement élevé.

\section{Conséquences des mesures sur l'emploi des couples}

Ces mesures ont eu un effet défavorable sur l'activité des femmes mariées ayant un conjoint qui travaille, comme le montrent un certain nombre d'études (voir tableaux 3 et 4). En effet, si l'activité de la seconde personne du couple fait entrer le ménage dans la phase de sortie de la mesure, cette dernière peut être incitée à quitter son emploi pour augmenter le montant des allocations perçues.

Eissa et Hoynes $(1998,2004)$ utilisent la méthode de doubles différences et constatent que la réforme de l'EITC de 1993 (large revalorisation du dispositif) a induit une baisse du taux d'emploi des mères mariées de 1,4 point (de 5 points pour les mères 
Tableau 3 : Résultats des études mesurant les effets de l'EITC sur l'activité des couples

\begin{tabular}{|c|c|c|c|}
\hline Étude & Période & Champ & Méthode d'évaluation et résultat \\
\hline \multirow[b]{2}{*}{ EISSA et HoYNES $(1998,2004)$} & \multirow[b]{2}{*}{ 1984-1996 } & mères mariées & $\begin{array}{l}\text { Doubles différences pour la réforme de 1993: } \\
\text { Effet sur le taux d'emploi des mères : }-1,4 \text { pts } \\
\text { (dont }-5 \text { points pour les mères avec au moins } 2 \text { enfants) }\end{array}$ \\
\hline & & $\begin{array}{l}\text { mères et pères en } \\
\text { couple }\end{array}$ & $\begin{array}{l}\text { Modèle structurel pour 1984-1996: } \\
\text { Effet sur le taux d'emploi : }-1 \text { pt pour les femmes (1) } \\
\text { dont : -2 points sur la phase de sortie de l'EITC } \\
\text { Effet faible positif pour les hommes } \\
\text { Effet global négatif }\end{array}$ \\
\hline Hotz, Mullin et Scholz (2005) & Réforme de 1993 & couples avec 2 enfants & $\begin{array}{l}\text { Doubles différences: } \\
\text { Effet positif sur le taux d'emploi : }+3,2 \text { pts par rapport } \\
\text { aux couples avec } 1 \text { seul enfant }\end{array}$ \\
\hline
\end{tabular}

(1) Principalement des mères, l'EITC n'étant ouvert aux familles sans enfant que depuis 1994.

Tableau 4 : Les évaluations du WFTC sur l'activité des différentes catégories de bénéficiaires

\begin{tabular}{|c|c|c|c|c|c|c|}
\hline \multirow{3}{*}{ Étude } & \multicolumn{5}{|c|}{ Effet sur le taux de participation (en points) } & \multirow{3}{*}{ Effet global sur l'emploi } \\
\hline & \multirow{2}{*}{$\begin{array}{l}\text { Mères } \\
\text { isolées }\end{array}$} & \multicolumn{2}{|c|}{ Femmes mariées } & \multicolumn{2}{|c|}{ Hommes mariés } & \\
\hline & & $\begin{array}{l}\text { Conjoint } \\
\text { chômeur }\end{array}$ & $\begin{array}{l}\text { Conjoint en } \\
\text { emploi }\end{array}$ & $\begin{array}{l}\text { Conjointe } \\
\text { inactive }\end{array}$ & $\begin{array}{l}\text { Conjointe } \\
\text { en emploi }\end{array}$ & \\
\hline \multicolumn{7}{|c|}{ Modèles structurels } \\
\hline $\begin{array}{l}\text { Blundell, DunCan, MaC Crae et } \\
\text { Meghir }(2000)^{(*)}\end{array}$ & $+2,2$ & $+1,3$ & $-0,6$ & $+0,4$ & $-0,3$ & +27500 \\
\hline GreGG, Johnson et ReEd $(1999)^{(*)}$ & $+1,9$ & $+1,8$ & $-0,8$ & $+0,5$ & $+0,05$ & +33000 \\
\hline $\begin{array}{l}\text { BREWER, DunCAN, SHEPHARD et } \\
\text { SuAREZ (2006) }\end{array}$ & $+5,1$ & & 0,6 & & & $\begin{array}{l}\text { WFTC seul }:+81000 \\
(+22000 \text { en tenant compte } \\
\text { des autres réformes })\end{array}$ \\
\hline \multicolumn{7}{|c|}{ Méthodes des doubles différences } \\
\hline LEIGH (2005) & $\begin{array}{l}\text { Ensemble } \\
\text { Effet globa } \\
\text { positif pou }\end{array}$ & $\begin{array}{l}\text { positif sur } \\
\text { les mères is }\end{array}$ & $\begin{array}{l}\text { participation } \\
\text { lées et celles }\end{array}$ & $\begin{array}{l} \\
+1 \text { point... } \\
\text { n couple }\end{array}$ & nt effet & \\
\hline
\end{tabular}

Note de lecture: le taux de participation des mères isolées a augmenté de 2,2 points de pourcentage suite à l'introduction du WFTC. Celui des femmes mariées dont le conjoint est en emploi a diminué de 0,6 point de pourcentage.

$\left.{ }^{*}\right)$ cités dans Blundell (2001).

avec deux enfants ou plus) par rapport aux femmes en couple sans enfant. Elles parviennent à la même conclusion en utilisant un modèle structurel simulé sur les trois réformes de l'EITC intervenues au cours de la période. Néanmoins, l'effet observé est plus faible qu'avec le modèle des doubles différences. La réduction du taux d'emploi est particulièrement marquée pour certaines catégories: le taux d'emploi des femmes en couple dont les revenus sont tels qu'elles se situent sur la partie décroissante du crédit d'impôt a baissé de plus de 2 points et leur nombre annuel d'heures de travail de $20 \%$. Cette baisse peut s'expliquer par le fait que le revenu du second conjoint peut faire entrer dans la phase de sortie du barème (l'EITC étant dépendant du revenu du ménage), phase où les taux marginaux d'imposition sont élevés et ainsi réduire les incitations des femmes ayant un conjoint occupant un emploi à entrer sur le marché du travail.

Hotz, Mullin et Scholz (2005) comparent les familles selon leur nombre d'enfants et concluent que le taux d'emploi des familles ayant plus de deux enfants a davantage augmenté que celui des familles ne comptant qu'un enfant(13). Ils cherchent ainsi à mesurer l'impact de l'extension de l'EITC de 1993, plus favorable aux familles de deux enfants ou plus et concluent que cette extension expliquerait les deux tiers du différentiel d'évolution des taux d'emploi entre 1993 et 1998 des deux types de familles. L'élasticité de l'offre de travail ainsi déduite est de l'ordre de 1,3 , et se situe plutôt dans la fourchette haute des estimations existantes.

Contrairement aux travaux sur l'EITC, les travaux sur le WFTC sont menés sur l'ensemble de la population. Ils mettent également en évidence pour l'essentiel un effet négatif sur l'activité du second conjoint

(13) Le taux d'emploi est défini dans cette étude de la manière suivante: si un membre de la famille au moins a un emploi, le taux d'emploi est égal à 1 . 
(voir tableau 4) à l'exception de l'étude de LeIGH (2005). Cet auteur conclut à un effet global positif du WFTC de l'ordre de +1 point sur le taux de participation. En isolant différentes «paires» de groupes dans la méthode de doubles différences (chaque paire associant un groupe de traitement à un groupe de contrôle), il observe également des effets positifs sur la participation et ce, quel que soit le groupe considéré (femmes seules, en couple, hommes seuls...). La hausse du taux d'emploi des femmes en couple peut paraître surprenante, car en contradiction avec la théorie comme avec les résultats obtenus sur l'EITC. En fait, LeIgH estime que le niveau élevé du WFTC (et notamment la baisse de la dégressivité) aurait suffi à compenser son effet revenu négatif sur les femmes dont le conjoint travaille déjà.

Comme dans le cas de l'EITC, les précédentes études utilisant la méthode des doubles différences se heurtent toutes au problème de l'identification de l'impact du WTFC par rapport aux autres mesures introduites ou modifiées à la même période. Les méthodes structurelles ont donc également été appliquées au Royaume-Uni pour isoler l'effet du WFTC (voir tableau 4).

Blundell, Duncan, Mac Crae et Meghir (2000) estiment des modèles structurels, tenant compte des coûts fixes liés à l'emploi et à la garde d'enfant, chaque coût dépendant du temps de travail associé à l'emploi recherché. Plus précisément, il s'agit de modèles de choix discret d'heures travaillées, estimés sur les familles avec enfants, qui différencient la situation des parents isolés ou en couple. Comme fréquemment dans ce type de modèle, les agents ont des préférences flexibles entre les heures et le revenu, qui dépendent de variables observables et inobservables. Pour les couples, le choix des heures est possible pour l'homme comme pour la femme (l'homme n'a cependant le choix qu'entre inactivité et temps complet). Un des résultats de cette étude est que l'introduction du WFTC aurait entraîné une baisse du taux de participation des femmes mariées avec un conjoint en emploi $(-0,6$ point). La modélisation utilisée est proche de celle de GregG, Johnson et Reed (1999); en revanche, les seconds ne modélisent pas la sortie de l'emploi et considèrent cette dernière comme invariante avant et après l'introduction de la mesure. Or, certains hommes peuvent en fait décider de se retirer du marché du travail suite à l'introduction du WFTC: c'est notamment ce qui explique la différence de résultats sur la participation des hommes mariés avec conjointe en emploi entre les deux études même si leurs mesures de l'effet global du WFTC sur l'emploi restent proches.

Les différentes études citées précédemment ont évalué l'effet du WFTC ex ante, c'est-à-dire tel qu'il était annoncé dans le budget 1998 et non tel qu'il a été effectivement mis en œuvre en 1999 incluant des augmentations de crédits d'impôt liés aux enfants à charge. De ce fait, leurs évaluations sont de moindre ampleur que celle de BREwER, Duncan, ShePhard et Suarez (2006), qui évaluent le WFTC tel qu'il a effectivement été introduit et tiennent compte des revalorisations intervenues par la suite jusqu'en 2002. Ils obtiennent ainsi un effet global sur l'emploi de +81000 . Lorsqu'ils prennent en compte les mesures mises en œuvre au même moment que le WFTC, l'effet est plus faible (+ 22000 emplois), ces mesures ayant plutôt contribué à faire diminuer le taux de participation des couples avec enfants.

$\mathrm{Au}$ final, les résultats sont moins favorables pour le WFTC que pour l'EITC au regard des montants affectés à chacune des mesures. Ceci peut s'expliquer d'une part par les interactions avec les autres dispositifs: le WFTC ayant été introduit en même temps que d'autres mesures allant dans le sens d'une réduction de l'offre de travail contrairement à l'EITC, et entrant de plus en compte pour le calcul des autres prestations ce qui, selon BLUNDELL et HoYNES (2001), diminuerait son efficacité sur l'emploi d'environ deux tiers. Aussi l'écart entre revenus d'assistance et revenus d'activité pour un emploi faiblement rémunéré est-il proportionnellement moins important au Royaume-Uni qu'aux États-Unis: d'après l'OCDE (2007a), l'impôt implicite (14) à la reprise d'une activité faiblement rémunéré (deux tiers du salaire du travailleur moyen), pour un parent isolé, serait en moyenne de $85 \%$ au Royaume-Uni, contre $46 \%$ aux États-Unis. En France, les gains à la reprise d'emploi sont demeurés encore plus limités avec la PPE, l'OCDE (2007a) estimant un taux d'imposition implicite au passage de l'inactivité vers l'emploi faiblement rémunéré de près de $100 \%$ pour un parent isolé(15). Le RSA, en étant plus élevé pour les faibles rémunérations, devrait contribuer à réduire ce taux marginal d'imposition, même si la plupart des autres prestations (allocations familiales, allocations logement...) restent incluses dans la base ressources du dispositif.

\section{Les conclusions des évaluations sur les heures travaillées et les salaires}

Peu d'études existent sur l'impact des mesures du type crédit d'impôt sur les heures travaillées. Globalement, elles concluent à des effets faiblement négatifs sur les heures travaillées des femmes mariées, le crédit d'impôt jouant de manière générale davantage sur la marge extensive (la participation)

(14) Cet «impôt implicite» mesure la diminution des prestations et l'augmentation de l'impôt induites par l'exercice d'un emploi, en pourcentage du revenu du nouvel emploi.

(15) Cette estimation a toutefois été réalisée avant les importantes revalorisations de la PPE. 
que sur la marge intensive (les heures travaillées). Eissa et LieBman (1996) observent un effet positif peu significatif sur les heures travaillées des mères célibataires et nul sur les mères célibataires peu qualifiées à l'aide d'un modèle de doubles différences. Pour Meyer et Rosenbaum (2001) qui utilisent un modèle structurel, l'effet de l'EITC sur les heures travaillées des individus en emploi est non significatif tandis que pour Scholz (1996), l'effet aurait été faiblement négatif. Au Royaume-Uni, GREGG et HARKNESS (2003) estiment par doubles différences que le WFTC n'aurait pas eu d'effet significatif sur les heures des individus en emploi à l'exception de ceux en dessous du seuil d'éligibilité (moins de 16 heures par semaine), qui auraient augmenté leur temps de travail pour bénéficier de la mesure.

EISSA et Hoynes (2005) estiment que cet effet négatif sur les heures travaillées est plus faible que celui que l'on pouvait attendre de la théorie et avancent plusieurs explications. D'une part, le modèle économique théorique n'est pas toujours vérifié : les salariés ne sont pas en mesure de choisir librement leurs heures travaillées. Eissa et Hoynes constatent que si certaines mères travaillent à temps partiel (principalement une partie de l'année), la majorité des mères célibataires travaillent à temps plein. D'autre part, un certain nombre de biais de mesure peuvent affecter les résultats. Tout d'abord, selon un résultat standard de la littérature sur l'offre de travail, l'élasticité de l'offre de travail est plus faible lorsqu'elle est estimée sur un échantillon de femmes qui travaillent plutôt que sur l'ensemble des femmes. L'élasticité des heures travaillées serait donc trop faible pour être appréhendée par des méthodes quasi expérimentales. Ensuite, les heures peuvent tout simplement être mesurées avec erreur. Les individus ayant des horaires variables peuvent aussi se tromper en calculant leurs heures moyennes travaillées sur l'année. Enfin, les individus peuvent ne pas connaitre parfaitement la forme de l'EITC. La plupart des salariés perçoivent l'EITC comme une somme forfaitaire avec leur avis d'imposition et ne connaissent pas nécessairement la forme exacte du dispositif. Ce résultat semble confirmé en France pour la PPE. En effet, selon une étude de la DARES et de la DREES, si les bénéficiaires connaissent bien les principes généraux de la PPE, en revanche, ses règles précises sont mal connues (DARES, 2008). $\mathrm{Au}$ final, les effets à attendre du RSA sur les heures travaillées seraient donc plutôt faibles.

Peu d'études existent sur l'impact des crédits d'impôts sur les salaires. On peut s'attendre, en premier lieu, à ce qu'un crédit d'impôt, augmentant le revenu individuel d'une personne en emploi, exerce une pression à la baisse sur le salaire versé par l'employeur. En effet, lorsque le crédit perçu est connu par l'employeur (comme c'est le cas du WFTC), ce dernier peut considérer que le crédit viendra compenser une éventuelle modération salariale. Azмат (2005) souligne le rôle de cet avantage infor- mationnel de l'employeur, et montre que le WFTC peut limiter la progression des salaires dans l'entreprise non seulement des salariés éligibles mais aussi des salariés non éligibles. D'une part, l'employeur partage avec les employés bénéficiaires du WFTC une part de leur prestation, ce qui se traduit par une moindre augmentation des salaires de ces derniers par rapport aux non bénéficiaires. L'employeur s'octroierait $35 \%$ du bénéfice du crédit d'impôt, d'où un effet négatif sur le salaire perçu par les hommes éligibles - l'effet étant non significatif pour les femmes (voir infra). D'autre part, selon Azmat, le WFTC est à l'origine d'externalités négatives. À qualification équivalente, les salaires d'un bénéficiaire et d'un non bénéficiaire du WFTC ne pouvant pas être trop éloignés, les salariés non éligibles sont également affectés par la modération salariale résultant de la mesure. Cette externalité est d'autant plus significative que la taille de l'entreprise considérée est importante et que la proportion de salariés éligibles au WFTC est élevée. En effet, si les salariés éligibles au WFTC sont peu nombreux, ils ne seront pas en mesure d'avoir une influence sur la politique salariale de l'établissement. Ces deux effets sont d'autant plus importants que la demande de travail est fortement élastique au coût du travail et leur ampleur varie en fonction du niveau de salaire. En particulier, l'effet direct sur le salaire des éligibles n'est pas significatif pour les salariés proches du salaire minimum, ce dernier constituant une borne inférieure en dessous de laquelle l'employeur ne peut fixer la rémunération. C'est ce qu'illustre l'effet mesuré par l'auteur sur la seule population féminine. De même, les salariés du haut de la distribution des salaires sont épargnés, étant donné la faiblesse du WFTC qu'ils perçoivent. Ce sont donc les salariés éligibles situés au milieu de l'échelle des salaires qui peuvent pâtir de la modération salariale.

Une deuxième étude s'intéresse aux effets du WFTC sur les carrières salariales par le biais de son impact sur la décision de se former du salarié. Le WFTC venant accroître le surplus tiré de la relation d'emploi, il peut ainsi conduire les salariés à davantage investir en formation générale (16). Selon Lydon et WaLKer (2005), en théorie, l'introduction du WFTC affecte les coûts et les bénéfices tirés d'une formation générale, induisant les salariés proches de l'exemption de la mesure à davantage se former, tandis que ceux qui bénéficient d'un WFTC plus conséquent ont moins intérêt à le faire. D'une part, une part du WFTC sert à financer la formation, diminuant son coût net. D'autre part, l'accroissement de salaire suite à la formation vient réduire le WFTC perçu par le salarié et ce, d'autant plus qu'il

(16) A priori pour une formation "généraliste», les coûts sont davantage répartis entre l'employeur et le salarié par le biais d'un salaire versé plus faible en début de poste que dans le cas d'une formation spécifique. En effet, une formation spécifique profitant plus à l'entreprise, ses coûts seront uniquement supportés par l'employeur. 
perçoit un montant de WFTC important. Ainsi, pour les salariés proches du seuil de sortie de la mesure, cette dernière diminue les coûts de la formation sans affecter les bénéfices tirés de cette dernière (la perte résiduelle du WFTC liée à l'augmentation de salaire suivant la formation est très faible). En revanche, pour les salariés éligibles durablement compte tenu de leur salaire, le WFTC réduit également les bénéfices tirés de cette dernière puisque les gains de la formation diminuent cette fois. Les auteurs comparent ensuite les trajectoires salariales des individus éligibles au WFTC à ceux anciennement éligibles ou non au $F C$ et constatent que ceux qui étaient anciennement éligibles et situés sur la partie décroissante du barème ont des trajectoires salariales moins favorables qu'auparavant (ces derniers étant moins enclins à voir leur nouveau crédit - plus élevé que l'ancien $F C$ - être amputé suite à une formation) tandis que ceux qui sont nouvellement éligibles au WFTC ont une croissance des salaires plus forte suite à leur éligibilité (l'effet «baisse du coût» d'une formation dû au WFTC l'emportant sur l'effet «baisse des bénéfices»).

Dans le cas de l'EITC, à la différence du WFTC, l'employeur ne connaît pas le montant perçu par son salarié, ni le fait qu'il soit éligible ou non. Ici, le canal d'action n'est pas la politique salariale de l'entreprise envers ses employés mais plutôt l'effet de la hausse de l'offre de travail sur le salaire des éligibles et des non éligibles. En effet, un crédit d'impôt efficace lié à la reprise d'une activité augmente l'offre de travail et donc, à court terme, le chômage. Ceci est susceptible d'exercer une pression à la baisse sur le salaire des individus concernés.

LeIGH (2007) utilise les différents suppléments d'EITC versés par les États pour mesurer l'effet d'une variation du montant d'EITC sur l'emploi et les salaires horaires versés. Il constate que les augmentations des taux d'EITC ont été associées à des hausses de l'emploi des salariés peu qualifiés ayant des enfants mais pas de celui des salariés sans enfants. En revanche, l'ensemble des salariés peu qualifiés, qu'ils aient des enfants ou non, a connu une baisse de salaire horaire (17). Cet effet est d'autant plus important que les individus considérés sont peu qualifiés: Leigh estime qu'une augmentation de $10 \%$ de la générosité de l'EITC aurait réduit de $4 \%$ le salaire horaire des non-diplômés et de $2 \%$ celui des titulaires d'un baccalauréat (l'effet étant nul sur celui des diplômés du supérieur). Cette hiérarchie proviendrait notamment du fait que l'EITC est plus généreux pour les salaires horaires faibles. Ces résultats peuvent être rapprochés de ceux de Rothstein (2005) qui montre que l'effet de l'EITC sur l'emploi diminue avec le niveau de salaire. En effet, l'effet d'appel sur les salariés les mieux rémunérés étant plus faible, la pression à la baisse de leur rémunération est plus limitée.

RothSTEIN (2008) exploite quant à lui l'expansion de l'EITC entre 1993 et 1995 par la méthode des doubles différences, afin d'en tirer des élasticités de l'offre et de la demande de travail différenciées selon le type de qualification. Ces élasticités sont ensuite intégrées dans un modèle d'équilibre entre offre et demande de travail faisant aussi intervenir un paramètre relatif au crédit d'impôt. Comme LeIGH (2007), l'auteur conclut que l'EITC tend à réduire le salaire finalement perçu par les salariés peu qualifiés (éligibles ou non à la mesure), une partie de ce salaire étant en fait captée par l'employeur.

D'autres travaux montrent que l'EITC augmente de toute façon le revenu des familles pauvres avec enfants, les gains associés à la reprise d'emploi l'emportant sur la baisse du salaire horaire (Neumark et Wascher (2001), Hotz et Scholz (2003)). Cependant, dans la mesure où les salariés avec et sans enfants perçoivent le même salaire, les salariés peu qualifiés sans enfants et donc non éligibles pourraient subir une perte de revenu quand l'EITC augmente. Il s'avère donc nécessaire de tenir compte des effets de la politique sur la population non éligible avant d'en modifier les paramètres.

Les études sur les salaires mettent donc en évidence qu'il faut différencier l'effet du crédit d'impôt sur les nouveaux embauchés et sur les travailleurs en place. Le risque est que les salaires proposés à l'embauche soient plus faibles (canal de l'offre de travail) et que les carrières salariales soient moins favorables (canal du partage des gains entre employeur et salarié). Néanmoins, un facteur important est celui de la connaissance ou non par l'employeur du montant du crédit ainsi que des salariés éligibles. Dans le cas du RSA, l'employeur n'étant pas directement informé des salariés susceptibles d'en bénéficier, c'est davantage par le canal de l'offre de travail que pourrait passer une éventuelle modération salariale qui affecterait plutôt les travailleurs à temps partiel rémunérés à un salaire horaire supérieur au Smic ainsi que les couples mono-actifs. De plus, la désincitation à se former induite par un crédit d'impôt plus élevé reste une limite à une mobilité salariale ascendante.

(17) Cette baisse s'explique par un effet de composition, les travailleurs récemment embauchés voyant leur salaire diminuer par rapport aux salariés en place. Ces derniers peuvent également être affectés par une certaine modération salariale. 
En conclusion, au vu des résultats des études anglo-saxonnes sur la participation, les heures travaillées et les salaires, on peut s'attendre à ce que le RSA, compte tenu de son ampleur pour les faibles rémunérations, ait un impact non négligeable sur la participation au marché du travail même si l'impact sur la marge intensive (en terme d'heures travaillées) risque d'être limité. Concernant les salaires, les effets modérateurs risquent surtout d'affecter les salariés à temps partiel, le salaire minimum constituant une borne inférieure, à moins que les employeurs ne réduisent la durée travaillée. Il peut cependant subsister un effet à plus long terme sur les carrières salariales, les employeurs étant moins incités à augmenter les salaires et les salariés à se former, en raison du crédit d'impôt.

De plus, l'efficacité d'une mesure dépend du contexte institutionnel dans lequel elle est s'inscrit: un crédit d'impôt est a priori plus efficace pour augmenter le revenu des personnes peu rémuné- rées et l'offre de travail dans les pays anglo-saxons caractérisés par un salaire minimum peu élevé et un système de taxes et de transferts moins développé qu'en France. Enfin, la France est un pays qui rencontre a priori davantage un problème de demande que d'offre de travail peu qualifié. Or, comme le souligne CAHUC (2002), l'efficacité d'un crédit d'impôt repose en partie sur la condition que l'augmentation de l'offre de travail permette effectivement de créer des emplois, condition qui n'est pas systématiquement satisfaite en France, notamment en période de crise. Face à ce problème de demande de travail peu qualifié, d'autres mesures peuvent être opérantes pour augmenter l'emploi, comme entre autres des allégements de cotisations patronales, des politiques actives de l'emploi ou encore des politiques de formation qui peuvent venir compléter le RSA. En effet, les premières mesures peuvent être efficaces à court terme pour favoriser le retour à l'emploi des travailleurs peu qualifiés tandis que la dernière est nécessaire à plus long terme (voir REMY (2006), OCDE (2007b), CREPON et al. (2007) sur ces différentes politiques). 


\section{Bibliographie}

Azmat G.Y. (2005), "The Incidence of an Earned Income Tax Credit: Evaluating the Impact on Wages in the UK", Mimeo, London School of Economics, November.

Blundell R., Brewer M., Reed H., Shephard A. (2005), 'Evaluating the market impact of Working Families' Tax Credit using difference-in-differences", Revenue Working Paper $\mathrm{N}^{\circ} 4$.

Blundell R., Duncan A., Mac Crae J., Meghir C. (2000), «The Labour Market Impact of the Working Families's Tax Credit», Fiscal Studies, Vol. 21, N 1, pp. 75-104.

Blundell R. Hoynes H. (2001), "Has In-Work Benefit Reform Helped the Labor Market?", NBER Working Paper $\mathrm{N}^{\circ} 8546$.

Blundell R., Walker I. (2002), “Working Families' Tax Credit: A Review of the Evidence, Issues and Prospects", Économie Publique, $\mathrm{n}^{\circ} 11,2002 / 2$.

Bonnefoy V., Buffeteau S., Cazenave M.-C. (2009), «De la prime pour l'emploi au revenu de solidarité active: un déplacement de la cible au profit des travailleurs pauvres», France portrait social, collection «INSEE Références », Paris, INSEE.

Brewer M. (2001), “Comparing In-Work Benefits and the Reward to Work for Families with Children in the US and the UK", Fiscal Studies, Vol. 22, № 1, pp. 41-77.

Brewer M. (2003), “The New Tax Credits”, Institute for Fiscal Studies, Briefing Note $N^{\circ} 35$.

Brewer M., Duncan A., Shephard A., Suarez M.J., (2006), 'Did Working Families' Tax Credit Work? The Impact of In-work Support on Labour Supply in Great Britain", Labour Economics, $N^{\circ} 13$, p. 699-720.

CAHuc P. (2002), «À quoi sert la prime pour l'emploi?», Revue française d'économie, vol. 16, $\mathrm{n}^{\circ} 3$, janvier.

Cochard M., Junod B., Arnaud F., Vermare S. (2008), «Les effets incitatifs de la prime pour l'emploi: une évaluation difficile», Économie et Statistique, n ${ }^{\circ} 412$, pp. 55-80.

Crepon B., Ferracci M., Fougere D. (2007), “Training the unemployed in France: How does it affect unemployment duration and recurrence?", IZA Discussion Paper, $\mathrm{N}^{\circ} 3215$, décembre.

DARES (2008), «La prime pour l'emploi: un dispositif bien connu dans son principe, peu dans ses modalités », Premières Synthèses, $\mathrm{n}^{\circ}$ 24-2, juin.

Delarue V. (2000), «Le Working Families' Tax Credit, un nouveau crédit d'impôt pour les familles à bas revenus au Royaume-Uni », Économie et Statistique, n 335 , pp. 47-61.

Eissa N., Hoynes H. (1998), "The Earned Income Tax Credit and the Labor Supply of Married Couples", NBER Working Paper $\mathrm{N}^{\circ} 6856$.

Eissa N., Hoynes H. (2004), "Taxes and the Labor
Market Participation of Married Couples: The Earned Income Tax Credit", Journal of Public Economics, Vol. 88, N 9-10, pp. 1931-1958.

Eissa N., Hoynes H. (2005), "Behavioral Responses to Taxes: Lessons from the EITC and Labor Supply", NBER Working Paper $\mathrm{N}^{\circ} 11729$.

Eissa N., Kleven H.J, Kreiner C.T. (2004), “Evaluation of Four Tax Reforms in the United States: Labor Supply and Welfare Effects for Single Mothers", NBER Working Paper $\mathrm{N}^{\circ} 10935$.

Eissa N., Liebman J., 1996, "Labor Supply Response to the Earned Income Tax Credit", The Quarterly Journal of Economics, Vol. 111, № 2, pp. 605-637, mai.

Francesconi M., Van Der KlaAuw W. (2004), "The Consequence of "In-Work" Benefit Reform in Britain : New Evidence from Panel Data", IZA Discussion Paper $\mathrm{N}^{\circ} 1248$.

Greep P., Johnson P., Reed H. (1999), "Entering Work and the British Tax and Benefit System", Institute for Fiscal Studies, London, March.

GregG, P., Harkness S. (2003), "Welfare Reform and Lone Parents in the UK", CMPO Working Paper Series $\mathrm{N}^{\circ} 03 / 072$

Hotz, V.J., Scholz J.K. (2003), “The Earned Income Tax Credit", in Moffitt, R. (Ed.), Means-Tested Transfer Programs in the US, University of Chicago Press.

Hotz V.J., Mullin C.H., Scholz J.K. (2005), “Examining the Effect of the Earned Income Tax Credit on the Labor Market Participation of Families on Welfare", California Center for Population Research, Working Paper, CCPR065-05, December 2005.

Laroque G., Salanie B. (2000), «Une décomposition du non-emploi en France», Économie et Statistique, ${ }^{\circ} 331$, pp. 47-66.

Leigh A. (2005), "Optimal Design of Earned Income Tax Credits: Evidence from a British Natural Experiment", Australian National University, Centre for Economic Policy Research, Discussion Paper $\mathrm{N}^{\circ} 488$.

LEIGH A. (2007), "Who Benefits from the Earned Income Tax Credit? Incidence Among Recipients, Coworkers and Firms", Mimeo, Australian National University, Research School of Social Sciences.

Looney A. (2005), "The Effects of Welfare Reform and Related Policies on Single Mothers' Welfare Use and Employment”, Federal Reserve Board, 2005-45.

LydON R., WALKER I. (2005), "Welfare to work, wages and wage growth", Fiscal Studies, Vol. 26, №3, pp. 335 370 .

Meyer B., Rosenbaum D. (1999), "Welfare, the Earned Income Tax Credit, and the Labor Supply of Single Mothers", NBER Working Paper, $\mathrm{N}^{\circ} 7363$. 
Meyer B., Rosenbaum D. (2000), "Making Single Mothers Work: Recent Tax and Welfare Policy and its Effects", National Tax Journal, Vol. 53, № 4.

Meyer B., Rosenbaum D. (2001), "Welfare, the Earned Income Tax Credit, and the Labor Supply of Single Mothers", Quarterly Journal of Economics, Vol. 116, № 3.

Neumark D., Wascher W. (2001), "Using the EITC to Help Poor Families: New Evidence and a Comparison with the Minimum Wage", National Tax Journal, Vol. 54, pp. 281-318.

OCDE (2007a), Prestations et salaires 2007: les indicateurs de l'OCDE, Paris, OCDE.

OCDE (2007b), «Activation des chômeurs : ce que font les pays», chapitre V, in Perspectives de l'Emploi, Paris, OCDE.

Paull G., Walker I., Zhu Y. (2000), “Child support reform: some analysis of the 1999 White Paper", Fiscal Studies, Vol. 21, N 1 , pp. 105-140.

Perivier H. (2007), «Dix ans après la réforme du Welfare américain », Lettre de l'OFCE, n 279.

Preston I.P., Walker I. (1999), "The Measurement of Living Standards in Labour Supply Models with
Nonlinear Budget Constraints", Journal of Population Economics, $\mathrm{N}^{\circ} 12$, pp. 343-361.

Remy V. (2006), «Quelle efficacité pour les politiques d'allégement de cotisations employeurs?», Travail et Emploi, $\mathrm{n}^{\circ} 105$, pp. 69-83.

Rothstein J. (2005), “The Mid-1990s EITC Expansion: Aggregate Labor Supply Effects and Economic Incidence”, Mimeo, Princeton University.

Rothstein, J. (2008), "The Unintended Consequences of Encouraging Work: Tax Incidence and the EITC", Mimeo, Princeton University.

Scholz J.K. (1996), "In-Work Benefits in the United States: The Earned Income Tax Credit", The Economic Journal, Vol. 106, № 434, p. 156-169.

Stancanelli E., (2007), "Evaluating the Impact of the French Tax Credit on the Employment Rate of Women", OFCE, Document de travail $n^{\circ}$ 2007-33, décembre 2007.

Stancanelli E., Sterdyniak H. (2004), «Un bilan des études sur la prime pour l'emploi», Revue de l'OFCE, $\mathrm{n}^{\circ} 88$, janvier. 